\title{
Estimativa da saturação de água do arenito Namorado na Bacia de Campos observando a dispersão dos dados dos perfis de poço
}

Gabriel Nacur \& Abel Carrasquilla, UENF/LENEP, Macaé - RJ

Copyright 2014, SBGf - Sociedade Brasileira de Geofísica

Este texto foi preparado para a apresentação no VI Simpósio Brasileiro de Geofísica, Porto Alegre, 14 a 16 de outubro de 2014. Seu conteúdo foi revisado pelo Comitê Técnico do VI SimBGf, mas não necessariamente representa a opinião da SBGf ou de seus associados. É proibida a reproducão total ou parcial deste material para propósitos comerciais sem prévia autorização da SBGf.

\section{Resumo}

Archie (1942) determinou a saturação de água em arenitos limpos através da medição da resistividade elétrica, constituindo-se esse num importante parâmetro na caracterização das reservas de hidrocarbonetos. Hingle (1959), Buckles (1965 e Pickett (1973) desenvolveram soluções gráficas para a Equação de Archie, que permitem uma rápida determinação da saturação de água, observando a dispersão dos dados. Assim, as saturações são estimadas uma vez feita a correção da porosidade do efeito da argilosidade, ficando demonstrado que a metodologia desenvolvida pode muito bem ser aplicada num cálculo rápido da saturação de água, porém, sem a determinação dos parâmetros da Equação de Archie.

\section{Introdução}

A saturação de água $\left(S_{w}\right)$ é um dos principais parâmetros a ser considerados na decisão de completar um poço para producir hidrocarbonetos ou abandoná-lo. Archie (1942), no seu trabalho pioneiro, mostrou a relação existente entre $S_{w}$ em arenitos limpos e a resistividade medida $\left(R_{t}\right)$ nas amostras de rochas, o que, posteriormente, foi estendido para perfis de poço (Schön, 2011). Em seguida, muitos outros autores realizaram estudos para desenvolver esquemas capazes de caracterizar $S_{w}$. Entretanto, nenhuma dessas abordagens pode ser considerada universal e ser usada em qualquer reservatório, pois é preciso estudar as características de cada reservatório para poder aplicar o modelo que melhor o explica (Ellis \& Singer, 2011)..

Existem muitos parâmetros petrofísicos que influenciam na estimativa de $S_{w}$, a saber: conteúdo de argila $\left(V_{s h}\right)$, porosidade $(\phi)$, permeabilidade $(K)$, resistividade da água da formação $\left(R_{w}\right)$, empacotamento dos grãos, etc. Uma maneira rápida e eficaz de estimar $S_{w}$ é usando gráficos crossplots de $R_{t}$ vs $\phi$, como foi feito por Hingle (1959), Buckles (1965), e Pickett (1973).

Utilizando a metodologia gráfica proposta por esses autores, calculamos a $S_{w}$ no reservatório turbidítico do Campo Namorado na Bacia de Campos, com a utilização de dados de perfis de poço (raios gama - $G R$, resistividade - $\mathrm{R}_{\mathrm{t}}$, neutrônico - $N P H I$, densidade - $R H O B$ e tempo de trânsito - DT) coletados nesse campo. O Arenito Namorado, consiste em depósitos de areias turbidíticas depositadas durante 0 Albiano -
Cenomaniano. De acordo com Bruhn (1998), a unidade sedimentar compõe a porção superior da Formação Macaé (Membro Outeiro), com o respectivo reservatório apresentando os seguintes atributos: área com fechamento do tipo misto, estrutural e estratigráfico (em torno de $23 \mathrm{~km}^{2}$ ); geometria externa lenticular/tabular definida por carbonatos da Formação Macaé; limite superior definido por folhelhos da Formação Ubatuba, limites laterais ao norte a ao sul por acunhamento, sudeste, noroeste e sudoeste por falhas com espessura média de $60 \mathrm{~m}$ (variando de 5 a $130 \mathrm{~m}$ ); a largura média de $4 \mathrm{~km}$ e comprimento mínimo de $9 \mathrm{~km}$ e máximo de $14 \mathrm{~km}$; a geometria interna é heterogênea, de baixo grau com estruturas primárias predominantes de arenito maciço e composição de arenitos arcosianos; a granulação é em média regular e o grau de arredondamento e esfericidade, em geral, é baixo.

\section{Metodologia}

Os estudos experimentais de Archie (1942) revelaram uma equação que relaciona quantitativamente $R_{t}$ (ohm.m) com $S_{w}$ (fração), $\phi$ (fração) e $R_{w}$ (ohm.m) em arenitos limpos, da seguinte forma:

$R_{t}=\frac{a R_{w}}{S_{w}^{n} \phi^{m}}$,

em que $a, m$ e $n$ são os coeficientes de de tortuosidade, de cimentação e de saturação, drespectivamente. Normalmente, $V_{s h}$ presente nos arenitos causa uma diminuição na $R_{t}$ da formação (Dalkhaa, 2005).

Hingle (1959) propus um método baseado em dados dos perfis $R_{t}$ e $\phi$, que permitem o cálculo de $S_{w}$ diretamente de um gráfico. Ele arrumou a Equação 1 da seguinte forma:

$$
\phi=\left[a\left(\frac{R_{w}}{S_{w}^{n}}\right)\right]^{\frac{1}{m}}\left(R_{t}\right)^{\frac{1}{m}} .
$$

Essa equação descreve um conjunto de retas que partem de uma origem comum, as quais representam valores de $S_{w}$ constante. A origem em comum é chamada de ponto da matriz com $\phi$ igual a zero. A reta $S_{w}$ igual a 1 (100\% saturada) pode ser desenhada partindo do ponto de matriz e passando pelos pontos que se localizam mais acima do gráfico de dispersão, cuja inclinação define $R_{w}$.

Pickett (1966) reorganizou a Equação de Archie, primeiramente isolando a $R_{t}$ como na Equação 1, e logo aplicou logaritmo nos dois lados dessa equação: 


$$
\log (\phi)=\frac{1}{m}\left[\log \left(a R_{w}\right)-n \log \left(S_{w}\right)-\log \left(R_{t}\right)\right]
$$

A Equação 3 representa uma reta em um gráfico de $R_{t}$ vs $\phi$ na escala logarítmica. A inclinação da reta definida por essa equação será igual à $-1 / m$ e intercepta a reta de $\phi=1$ no valor numericamente igual a $a R_{w}$. Se assumimos que $a=1$ pode-se facilmente calcular $R_{w}$. As outras retas com $S_{w}$ constantes serão paralelas à reta $S_{w}=1$, a distância perpendicular entre estas retas de $S_{w}$ constantes irão depender da parcela $n \log \left(S_{w}\right)$ presente na Equação 3. Como vantagem, o gráfico de Pickett permite o cálculo de $m, R_{w}$ e avaliar qualitativamente $S_{w}$ de acordo com a proximidade das retas de saturação de água constantes. Mas, com esse método é necessário assumir ainda valores para $n$ e $a$.

Uma vez $S_{w}$ é conhecida, Buckles (1965) indicou a forma de calcular o conteúdo volumétrico de água no reservatório $(B V W)$, através da relação:

$$
B V W=\phi S_{w} .
$$

Um mínimo de $B V W$ implica em saturação de água irreducível $\left(S_{\text {wirr }}\right)$, quando há produção de pouca e nehuma água. Quando se plota $S_{w} v s \phi$ em escala linear ou logarítmica, mostra-se $B V W$ sem ter que fazer cálculos.

\section{Resultados}

A Figura 1 mostra os perfis $G R, R_{t}, N P H I, R H O B$ e $D T$ coletados no poço NA02 do Campo Namorado, num intervalo entre 2.980 até $3.170 \mathrm{~m}$ de profundidade. Nesse poço, o óleo foi detectado no intervalo 3.041 $3.054 \mathrm{~m}$ durante a perfuração pelo estudo das amostras de calha presentes no fluido de perfuração, quando uma presença constante de hidrocarbonetos foi registrada, podendo ser caracterizado como a zona produtora. Qualitativamente podemos notar esse possível reservatório de hidrocarbonetos no intervalo 3.020 até 3.060 metros de profundidade devido a valores baixos de $G R$ e $R_{t}$ alta (retângulo vermelho).

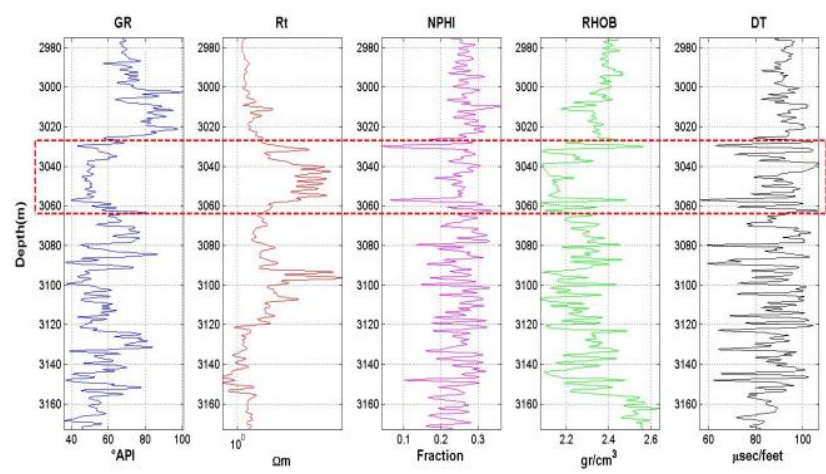

Figura 1. Perfis no poço NA02.

Enseguida, após corrigir a porosidade dos efeitos da argilosidade (Krygowski \& Cluff, 2012) para esse reservatório, fizemos o gráfico de Hingle, o qual aparece na Figura 2, a qual mostra círculos vermelhos com cruz no centro indicando os centros dos agrupamentos calculados com a técnica K-means (Forgy, 1992). Os dois agrupamentos inferiores apresentam uma porosidade de aproximadamente 0,26 , quanto que o outro agrupamento apresenta uma porosidade um pouco menor, de aproximadamente 0,23 , parecendo haver uma diminuição com a profundidade. Observam-se três áreas principais: a primeira, do círculos amarelos, é uma zona com pouca ou nenhuma $S_{w}$, entre 0,1 até 0,0 , que corresponde ao óleo dentro do reservatório, com valores de condutividade de 0.15 mhos.m. A segunda, a dos círculos azuis, é a zona de transição água - óleo com $S_{w}$ entre 0.1 e 0.3 e condutividade de 0.30 mhos.m. A terceira zona, que corresponde aos círculos verdes é aquela com $S_{w}$ com valores entre 0,4 até 1,0 e condutividade de 0.50 mhos.m.

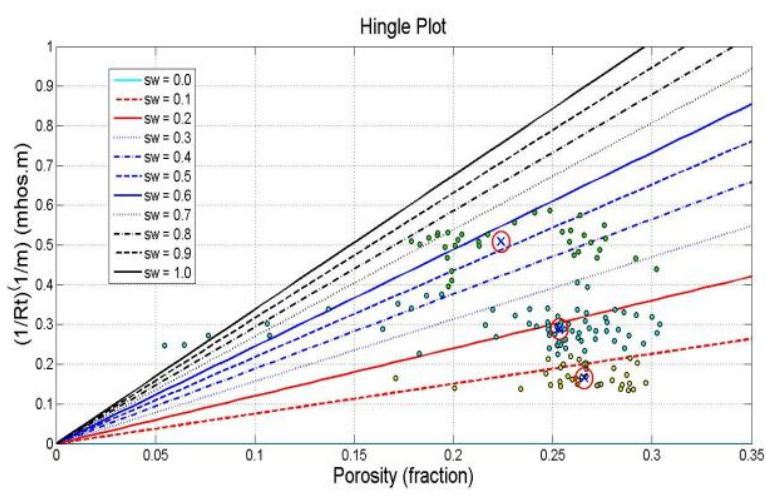

Figura 2. Gráfico de Hingle para o reservatório do poço $\mathrm{Na} 02$.

No gráfico de Pickett (Figura 3), os três centros de agrupamentos indicam uma porosidade aproximada de 0,27 para todo o reservatório. Neste caso, os círculos amarelos indicam a zona com $S_{w}$ acima de 0,2 , os verdes a zona de transição com $S_{w}$ entre 0,2 e 0.1 e os azuis a zona de óleo com $S_{w}$ menor que 0.10 , com resistividades baixa (10 ohm.m), média (65 ohm.m) e alta (450 ohm.m), respectivamente.

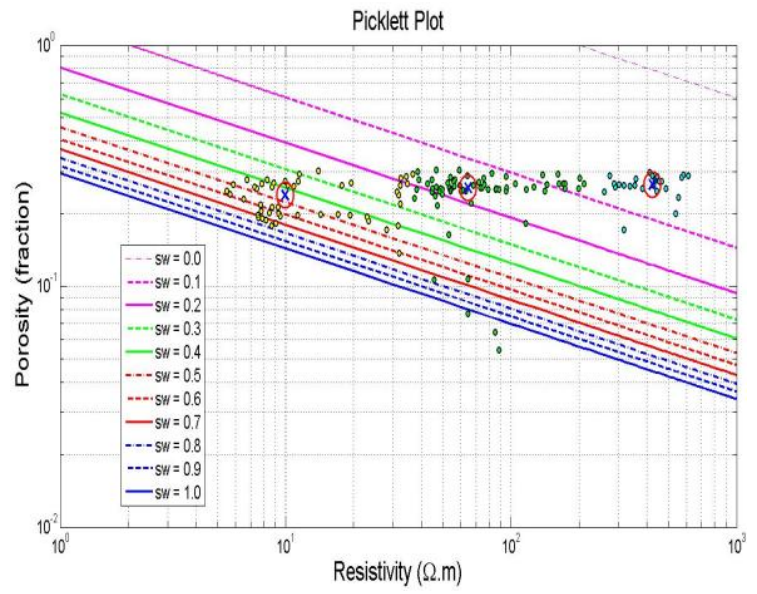

Figura 3. Gráfico de Pickett para o reservatório do poco $\mathrm{Na} 02$.

No caso do gráfico de Buckles (Figura 4), os dosi primeiros agrupamentos indicam uma seção do reservatório com porosidade aproximada de 0,27 e 
uma outra com 0,20 . Novamente as três zonas antes mostradas são muito evidentes neste caso: a amarela representa a seção do óleo com $B V W$ entre 0,00 e 0,08 $\left(S_{w}=0,13\right)$. A verde é a zona de transição com $B V W$ entre 0,08 e $0,12\left(S_{w}=0,40\right)$, e, finalmente, a azul, a zona de óleo com com $B V W$ maior que $0,12\left(S_{w}=0,63\right)$. Os valores menores de $S_{w}$ mostram uma zona com m valor aproximado de 0,04, que corresponde ao valor da saturação de água irredutível $\left(S_{\text {wir }}\right)$.

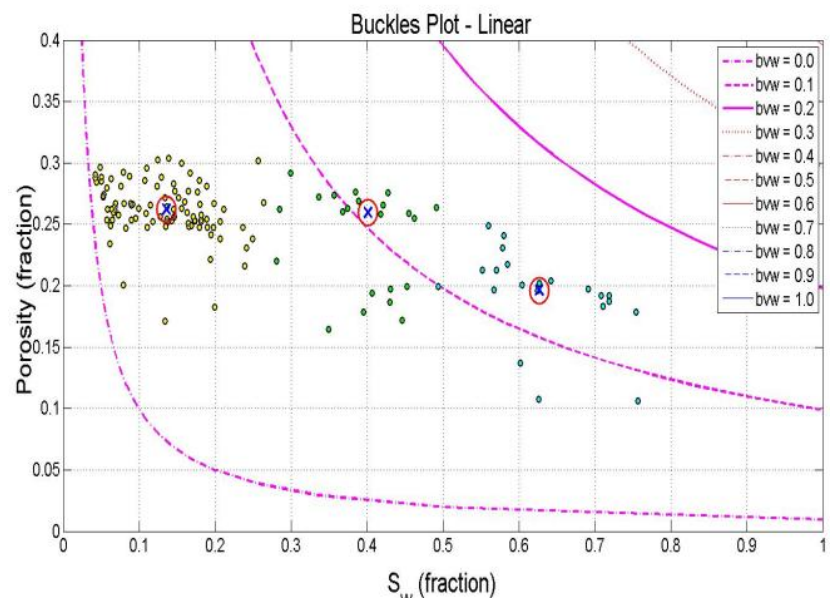

Figura 4. Gráfico de Buckles para o reservatório do poço $\mathrm{NaO2}$.

\section{Conclusões}

A $S_{w}$ do poço NA02 do Campo de Namorado, na Bacia de Campos - Sudeste do Brasil, foi estimada através de dados de perfis de poço, considerando um meio poroso para arenitos limpos como a Equação de Archie. Os gráficos croosplots de Hingle (1959), Pickett (1973) e Buckles (1965) apresentam a vantagem de nos dar uma visão espacial dos dados, ao separar os valores de $S_{w}, S_{w i r}, R_{t}, B V W, \phi$ nas diferentes zonas de óleo, transição e aquífero dentro do reservatório, mas, não fornecem os valores dos parâmetros $a, n, m e R_{w}$ da Equação de Archie. Ou seja, a não determinação exata dos parâmetros da Equação de Archie pode levar a uma estimativa errada de $S_{w}$, e, portanto, as reservas de hidrocarbonetos $\left(S_{o}=1-S_{w}\right)$. Assim, métodos quantitativos de cálculo desses parâmetros são mais aconselhados.

\section{Agradecimentos}

Agradecemos à ANP por ceder os dados utilizados neste trabalho, à Petrobras pelo financiamento de projeto de pesquisa e ao UENF/LENEP pela infraestrutura computacional.

\section{Referências}

- Archie, G. 1942. The electrical resistivity log as an aid in determining some reservoir characteristics. Transactions of the American Institute of Mining, Metallurigical and Petroleum Engineers, 146, 54-62.

- Bruhn, C. 1998. Deep-water reservoirs from the eastern Brazilian rift and passive margin basins.
Extended abstracts, AAPG International Conference \& Exhibition, Rio de Janeiro, $187 \mathrm{p}$

- Buckles, R.S. 1965. Correlating and averaging connate water saturation data. Journal of Canadian Petroleum Technology, v. 9, no. 1, p. 42 - 52.

- Dalkhaa, C. 2005. Study of modeling of water saturation in Archie and non-Archie porous media. Master Thesis, Middle East Technical University, Temmuz, $112 \mathrm{p}$.

- Ellis, D. \& Singer, J. 2011. Well logging for earth scientists. Springer, $699 \mathrm{p}$.

- Forgy, E. 1965. Cluster analysis of multivariate data: efficiency versus interpretability of classifications. Biometrics, v. 21, p. 768-769.

- Hingle, A. 1959. The use of logs in exploration problems; Paper presented at the SEG $29^{\text {th }}$ International Annual Meeting, Los Angeles.

- Krygowski, D. \& Cluff, R. 2012. Pattern Recognition in a Digital Age: A gameboard approach to determining petrophysical parameters. Poster Session, AAPG ACE Meeting.

- Pickett, G. 1973. Pattern recognition as a means of formation evaluation. SPWLA $14^{\text {th }}$ Annual Logging Symposium, Paper A.

- Schön, J. 2011. Physical properties of rocks: a workbook. Elsevier, Amsterdam, 479 p. 